\title{
A Convolutional Neural Network for Dental Panoramic Radiograph Classification
}

\author{
James Faure \\ Department of Industrial Engineering \\ Stellenbosch, South Africa \\ jamesfaure@icloud.com
}

\author{
Andries Engelbrecht \\ Department of Industrial Engineering, and Computer \\ Science Division \\ Stellenbosch, South Africa \\ engel@sun.ac.za
}

\begin{abstract}
Radiographs are X-rays of the craniofacial area, used for orthodontic diagnosis and treatment planning. Analysis of radiographs is a manual process. Public medical centers in developing countries such as South Africa experience a bottleneck in the analysis of these radiographs, due to excessive numbers of patients and severe shortages in orthodontic radiologists that serve at these public medical centers. Access to dental diagnostics is therefore becoming an ever-increasing problem in rural communities.

This paper reports on the first phase of a framework to automate the analysis of panoramic radiographs, which are X-rays of the frontal croniofacial area. This first phase automates the process to predict whether a captured panoramic radiograph is workable or not, i.e. whether automated analysis of the X-ray can proceed. A convolutional neural network $(\mathrm{CNN})$ is trained on a large set of panoramic radiographs, and results show that the prediction accuracy of this $\mathrm{CNN}$ is very good in identifying workable radiographs.
\end{abstract}

\section{CCS CONCEPTS}

- Computing methodologies $\rightarrow$ Convolutional neural network; Image classification; • Image analysis $\rightarrow$ Panoramic radiographs; Digital images; Pixellation.

\section{KEYWORDS}

Panoramic radiograph, Orthodontic radiology, Convolutional neural network, Image Analysis

\section{ACM Reference Format:}

James Faure and Andries Engelbrecht. 2021. A Convolutional Neural Network for Dental Panoramic Radiograph Classification. In ISMSI '21: 2021 5th International Conference on Intelligent Systems, Metaheuristics \& Swarm Intelligence (ISMSI '21), April 10-11, 2021, Victoria, Seychelles. ACM, New York, NY, USA, 6 pages. https://doi.org/10.1145/3461598.3461607

\section{INTRODUCTION}

Analysis of the structure of the mouth can be a complicated and time-consuming task for any dentist or orthodontic radiologist. A common procedure used to complement the clincial examination of

Permission to make digital or hard copies of all or part of this work for personal or classroom use is granted without fee provided that copies are not made or distributed for profit or commercial advantage and that copies bear this notice and the full citation on the first page. Copyrights for components of this work owned by others than ACM must be honored. Abstracting with credit is permitted. To copy otherwise, or republish, to post on servers or to redistribute to lists, requires prior specific permission and/or a fee. Request permissions from permissions@acm.org.

ISMSI '21, April 10-11, 2021, Victoria, Seychelles

(c) 2021 Association for Computing Machinery.

ACM ISBN 978-1-4503-8967-9..\$15.00

https://doi.org/10.1145/3461598.3461607 a patient is to take a panoramic radiograph of the region of interest. A lateral radiograph, referred to as a cephalogram, provides a profile of the skull and soft tissues, and is used to assess the relation of the teeth in the jaws, the relation of the jaws to the skull and the relation of the soft tissues to the teeth and jaws. A panoramic radiograph (refer to Figure 1), provides a frontal profile of the jaws, teeth and soft tissues. Evaluation of these radiographs involves individually checking each tooth to assess whether it is present and to determine if any anomalies occur.

Analysis of these radiographs is a manual process, which becomes a time consuming task for public medical centers in developing countries. Due to the time-consuming nature of reporting and analysis of panoramic radiographs, and the shortage of qualified orthodontic radiologists, defects in many of these radiographs are left undiagnosed. In a public hospital in the Western Cape, South Africa, the screening centre has on average 120 patients per day with only one or two orthodontic radiologists available, resulting in only around 30 patients getting adequate reporting. The radiographs are screened, and if there are no significant findings, no report is compiled. There are also medico-legal implications if a disease or lesions are not reported.

In order to address this problem, analysis of these radiographs, and subsequent reporting, can be automated. The solution is to build a model that facilitates automatic reporting on radiographs during the screening process. This paper addresses the first phase of an automated system for radiograph analysis and reporting. For this phase, radiographs are received and a convolutional neural network (CNN) is used to predict if radiographs are of sufficient quality for automated analysis and are, specifically, panoramic radiographs.

The rest of the paper proceeds as follows: Section 2 provides background in support of this paper. Section 3 discusses the image preprocessing techniques that have been applied on all of the radiograph images that have been used for training and testing the CNN. Section 4 provides detail on the $\mathrm{CNN}$ architecture, its training, and presents results to illustrate the prediction accuracy of the CNN.

\section{BACKGROUND}

There are two important elements to understand before training an image classification model. The first being the images that exist in the dataset, and the second being the machine learning algorithm that will be used to model the dataset. For the purposes of this research, the dataset consists of radiographs, and the best algorithm for image classification is CNNs. Therefore, this section provides background on both radiographs and CNNs. 


\subsection{Introduction to X-Ray analysis}

Radiographs, more commonly known as X-rays, are the best known medical diagnostic images. In 1895, Róentgen accidentally discovered X-rays [3]. At the time Röentgen was observing the effects of cathode rays in an airtight tube. Under a high voltage, the tube produced a fluorescent glow. Röentgen then shielded the ray with heavy black paper and noticed that a material located a few feet away started to glow [2]. Röentgen then used this light to observe his hand, and to his surprise, he saw his bones.

Capturing of digital radiographs require an X-ray machine, a sensor and a central processing unit. The sensor captures the signal (visual information) which is then processed by and algorithm and converted to a visible image. Each pixel on the visible image is represented as a particular shade of grey. The shade of grey relates to the level of radiation, with black being $0 \%$ and white being $100 \%$ radiation. On a radiograph, denser objects such as teeth or bones are closer to $100 \%$ radiation, while soft tissue is closer to $0 \%$ radiation.

\subsection{Convolutional neural networks}

A CNN is a class of deep neural networks that are applied to analyse image prediction tasks. The algorithm processes grid-structured inputs, which have strong spatial dependencies in local regions of the grid [1]. A CNN takes input images and assigns importance to features and objects. The primary purpose of the CNN is to find features and to put them in a feature map, while still preserving the pixellation. The following sections discuss CNNs.

2.2.1 Introduction of the basic structure. A CNN has four classes of layers, namely convolutional layers, rectified linear unit (ReLU) layers, pooling layers, and fully connected layers. Each layer in the network has a three-dimensional grid structure, which has height, width and depth. The depth of the layer must not be confused with the depth of the entire network. The layers work in an interlinked fashion to process the input image into a predicted output. A binary classification function predicts the output as part of the final step of a CNN. For the remainder of this paper, the structural units of layer $i$ have a height of $H$, width of $W$, and depth of $d$.

2.2.2 Convolutional layer. The convolutional layer performs a process called a convolution operation. The convolution operation takes a grid-structured set of weights and a similar grid-structured set of inputs from different spatial localities in the input image and performs a dot-product operation [1]. The convolution operation places a filter $K$ at a spatial location of image $I$ (or hidden layer i) to generate a feature map $I^{*} K$. The depth of the layer conveys the different channels in each layer, whether it is the number of primary colours or the number of feature maps in the hidden layers.

A filter is constructed out of parameters that are organized into sets of three-dimensional structural units. The dot-product operation applies the parameters in the filter to a matching grid on the input image [1]. The filter slides across that receptive field (i.e. the image), and in each position, the filter multiplies the value of the filter by the original values of the pixels. The multiplications are summed up, creating a single number, which represents the area just filtered. If filter $F_{q} \times F_{q} \times d_{q}$ is applied to layer $H_{i} \times W_{i} \times d_{i}$, the resultant layer has a new dimension of $\left(H_{i}-F_{q}+1\right) \times\left(W_{i}-F_{q}+1\right) \times d_{i+1}$, where $d$ changes to the number of parameters of the applied filter
$F$ [1]. The spatial arrangement of pixel values, obtained from the output of a filter, is called a feature map.

The feature map is made up of an array of numbers that represents the depth of a filter. The CNN learns the values of the filters during training. By increasing the number of filters of a particular layer, more feature maps result in the following layer. A more significant number of filters is required to capture a wide variety of possible features. The features are eventually combined to create a final image. A feature map is smaller than the original image and therefore more accessible and faster to deal with. The process loses some information; nevertheless, features can still be detected. The model does not detect every pixel, but instead detects features made up of pixels.

Information is lost along the borders of an image (or layer) due to the difference in size between layer $i$ and $i+1$. Padding is a technique used to add pixels around the feature map [1]. The value of these pixels is set to zero in order for them to have no effect on the dot-product operation of the next filter [1].

Strides are another essential part of the convolution operation. In some instances, it is not necessary to perform a convolution at every spatial position in the layer [1]. Strides reduce the spatial variance of the image (or hidden layer).

2.2.3 Rectified linear unit layer. The ReLU layer applies an activation function to the feature maps. Images are non-linear by nature, and the ReLU activation function increases the non-linearity of the network. The function removes all negative pixel values from the activation map by setting them to zero, hence changing the negative pixel values to black. The result of this process is an activation layer which usually follows every convolutional layer. The ReLU layer trains the network faster, without hindering the generalisation accuracy. The ReLU activation function is given by $f(x)=\max (0, x)$, where the output is zero when $x<0$ and a linear function when $x \geqslant 0$.

2.2.4 Pooling layer. The pooling layer is a two-dimensional grid of size $P_{q} \times P_{q}$. The pooling layer operates on an activation map to create another feature map with the same depth, but smaller spatial dimensions. Strides that are greater than one are commonly used to reduce the spatial variance of the layer. For image (or layer) $L_{\mathrm{q}} \times B_{\mathrm{q}}$ that has a pooling layer with a stride of $\mathrm{S}_{\mathrm{q}}$ applied to it, the resultant layer is $\left(\left(L_{\mathrm{q}}-P_{\mathrm{q}}\right) / S_{\mathrm{q}}+1\right)\left(\left(B_{\mathrm{q}}-P_{\mathrm{q}}\right) / S_{\mathrm{q}}+1\right)[1]$.

The pooling layer adds flexibility to the $\mathrm{CNN}$ by giving the model spatial variance. A pooling layer is needed to change the spatial size to a small uniform size [1]. The model is trained to recognise the same features in many different forms of the images, hence dealing with overfitting. Pooling layers are interleaved with convolutional and ReLU layers.

The two most common types of pooling are maximum pooling and average pooling. Maximum pooling returns the maximum value of the activation maps. Average pooling returns the average value of a pool on the activation maps.

2.2.5 Fully connected layer. Finally, the fully connected layer (FLC) is added onto the end of the network. The primary purpose of the FLC is to combine features into more attributes, which help to predict classes and objects with high accuracy. The final spatial 
layer contain features that are connected to each hidden state in the first FLC [1].

\subsection{Combining the layers to train the $\mathrm{CNN}$}

Next, the layers are combined in an alternating pattern to build the predictive model. The model is trained over many itereations on the training dataset. Through the training process, the model learns the pixellation of the images.

2.3.1 The architecture. The convolutional layer is directly followed by a ReLU activation function. Next, max-pooling is applied to the convolutional layer. The alternation between these two layers is usually repeated a certain number of times before a connected layer represents the final feature maps. The depth of the network is directly proportional to the number of alternations between convolutional, ReLU and pooling layers. Finally, the classification layer uses a sigmoid function to predict the model outputs.

2.3.2 The training process. Once the final architecture of the network is structured, training must begin. A CNN uses a backward propagation algorithm. The error derivatives between layers $i$ and $(i+1)$ are computed and multiplied with the transpose of the forward propagation matrix between layers $i$ and $(i+1)$ [1]. The backward propagation happens at each layer until the weights on each output neuron are fully optimised.

2.3.3 ResNet. The ResNet [1] deep network architecture was the first model to perform at a human level classification. The architecture has 152 layers. Acquiring such a depth required important innovations of the model's structure. A large number of operations in deep layers causes the gradient flow of feature maps to be impeded [1]. Consequently, a new technique called skip connections was developed. A skip connection introduces an iterative process of feature engineering, where information can be copied between layers. Backwards propagation provides a useful link between layers for propagating gradients backwards using skip connections [1]. Each layer has a padded filter of stride one, which ensures that the spatial size of the outputs does not change between layers.

The ResNet model is commonly found as the backbone algorithm of most machine learning packages.

\section{DATASET PREPARATION}

The raw data must be analyzed to develop a deeper understanding of the images. The images dataset provided forthis reseach contained unlabelled images, and therefore was an unsupervised dataset. The X-rays therefore first went through a labelling process to produce a supervised dataset. This process added to each image a label, either "orkable" or "unworkable".

\subsection{The dataset}

The dataset consists of 2500 panoramic radiographs. Figure 1 shows four examples of images taken from the dataset. The original images are in tagged image file format (TIFF), which means that the file sizes are close to $9 \mathrm{MB}$ each.

The dataset is analyzed by selecting a small subset of images and manually exploring the features that exist within each X-ray. The results of the analysis show that there are four broad categories of radiographs that can be classified into two main classes. Figure
1 shows the four types of images, which are classified as either "workable" or "unworkable". Figure 1a shows an X-ray that can be used for diagnosis by a dentist, hence the image is classified as "workable". Figure 1b displays a blurry image that cannot be used with ease for diagnosis by a dentist, hence this image is classified as "unworkable". Figure 1c shows a cephalometric radiograph, a different type of X-ray used by dentists. Although this is a common tool for diagnosing patients, the image is classified as "unworkable" for the specifics of this project. Figure $1 \mathrm{~d}$ shows an X-ray that produced extreme noise when captured, hence the image is also classified as "unworkable".

Despite having unworkable images, the larger portion of the dataset remains workable. Because the reporting process is automated, it is necessary to detect if an X-ray is workable or not. After understanding the dataset, it is evident that due to human processing error creating unworkable images, an image classification algorithm must be built to separate the workable images from the unworkable images.

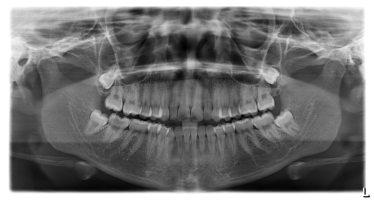

(a) Panoramic radiograph

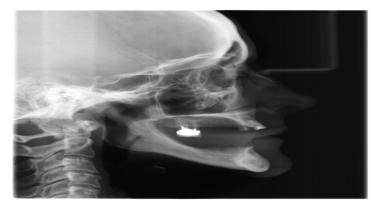

(c) Cephalometric radiograph

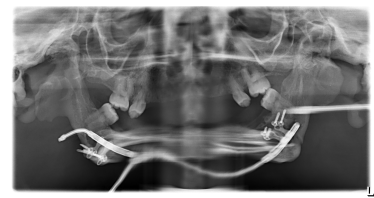

(b) Blurry panoramic radiograph
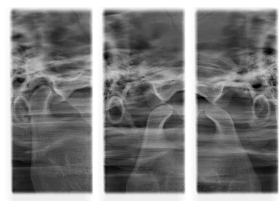

(d) Noisy radiograph
Figure 1: A small subset of images taken from the dataset.

\subsection{Image Preprocessing}

All labelling tools require images to be in either portable network graphics (PNG) or joint photographic experts group (JPEG) format. The file sizes of a TIFF image is too large for efficient image processing, therefore the first data manipulation technique is to convert the TIFF images to PNG. The files are imported into a Jupyter notebook and converted to PNG images using a python script. Figure $2 \mathrm{a}$ and $2 \mathrm{~b}$ show the process followed to convert the initial dataset of TIFF files into smaller, more compressed PNG files. An important observation is that the quality of the images is not impeded.

In the conversion to PNG, the orientation of the images changes. The capital letter ' $L$ ' in the bottom right corner of Figure 2a denotes the left-hand side of the patient's mouth. This is used to check the orientation of the image. A Python script is used for the manipulations to correct the orientation. The first translation from Figure $2 \mathrm{~b}$ to Figure $2 \mathrm{c}$ requires the image to be rotated $180^{\circ}$, placing the ' $\mathrm{L}$ ' in the bottom left corner of the image. The final translation from Figure $2 \mathrm{c}$ to Figure $2 \mathrm{~d}$ is a correction in the form of a horizontal flip along the $y$-axis of the image. Comparing Figure 2a to Figure $2 \mathrm{~d}$ shows that the image orientation is now correct. 
The conversion and manipulation process is repeated for the entire dataset. Each image is compressed from an initial 9MB TIFF file to around $2 \mathrm{MB}$ as a PNG file, without impairing the quality of the pixellation. The compression results in lower computational expenses, quicker processing times and cheaper storage during future applications.

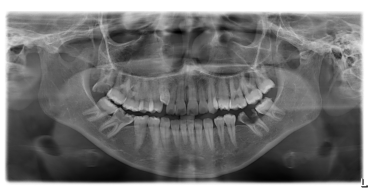

(a) Initial image as a TIFF

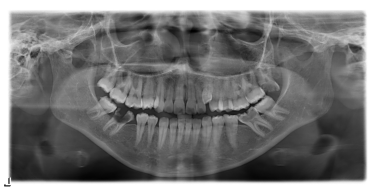

(c) Rotated $180^{\circ}$

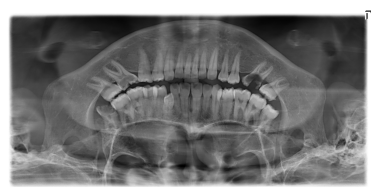

(b) First conversion to PNG

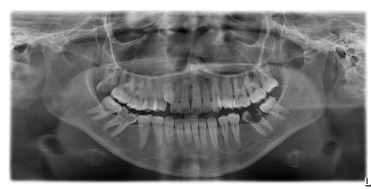

(d) Horizontal flip to final image
Figure 2: Data manipulation from a large TIFF file to a compressed PNG file.

\subsection{Dataset Labelling}

A patient who has had an X-ray done expects to be diagnosed. In order for an X-ray to be a usable diagnostic tool, it must be clear and readable to the dentist or radiologist. The same applies to the image classification model. In order for the pixellation of each tooth or feature to be understood, the image must be clear and concise.

As discussed in section 3.1, the complete dataset is made up of two classes of images. Specific X-rays in the dataset are unclear, hence need to be removed and avoided in the future. Therefore, it is necessary to build an image-classification model to be able to differentiate between "workable" and "unworkable" images.

Each image in the dataset is manually checked to determine its workability status. The labelling process is tedious and lengthy. Often, large datasets are labelled over many days. Consequently, a lean labelling strategy is used for the annotation process, by first labelling a small subset of images, then testing the model for accuracy. If the model accuracy is not adequate, more images can be labelled and added to the training dataset.

For the subset, 635 images were separated into the two classes: 501 were "workable" images and 134 "unworkable" images. The images are separated into three subsets, namely the training, validation and testing subsets. Figure 3 shows the class distribution of these sets. If the model accuracy is insufficient, it will be simple to add more images to each subset and rerun the algorithm.

\section{IMAGE CLASSIFICATION}

After labelling the dataset into either "workable" or "unworkable" classes, a model must be built and trained to learn each labelled class. The training process involves preparing the dataset, configuring the model and finally using the training data to set parameters. A second dataset, called the validation subset, is used to evaluate

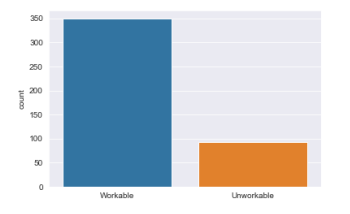

(a) "Workable" and "unworkable" images of the testing dataset.

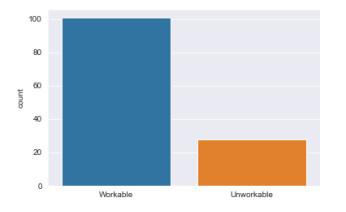

(b) "Workable" and "unworkable" images of the validation dataset.

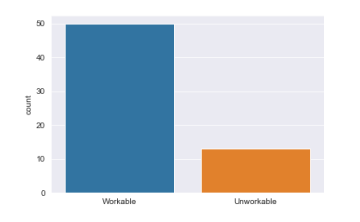

(c) "Workable" and "unworkable" images of the testing dataset.

Figure 3: The image splitting process between the training, validation and testing dataset.

the performance of the model. Finally, evaluation metrics are used to determine the performance of the model. The model is built in python using a jupyter notebook and the TensorFlow machine learning libraries.

\subsection{Building the model}

The first step of the machine learning process is to choose an appropriate model. As discussed in section 2.2, the best model for image classification is a CNN. The model learns the pixellation of the training images and make predictions on a similar subset of images.

The image classification model is trained using Keras, which is an industry-strength framework used to train and scale large cluster GPUs. Keras is built on top of Tensorflow 2.0, a machine learning platform hosted by Google. The Keras applications are built with a variety of different ResNet implementations that can be constructed with a different number of layers.

\subsection{Training the model}

Once the dataset is ready, the model is built for training. In order to build a successful model, the dataset goes through final preparations and the model is configured according to user specifications.

4.2.1 Preparing the dataset. As mentioned in section 3.3, the dataset must be split into three subsets, namely training, validation and testing datasets. The training set consists of 447 images and is used to train the prediction model. The validation set has 127 images and is used to validate the accuracy of the trained model. The validation set is crucial because it indicates whether the dataset needs any improvements, or if model parameters need any adjustments. Finally, the testing set contains 61 images and is used in a similar way to the validation set, except that actual predictions are made to estimate generalization performance. This split means that the training set holds $70 \%$ of the images, the validation set has $20 \%$, and 
the testing set the remaining $10 \%$. As discussed in section 3.3 , the split was enforced in the labelling process when the two classes were divided into their respective subsets.

Each image is stored as an array of pixel values with corresponding target labels. In essence, this is what makes the dataset a supervised dataset. The target label is a binary value of 0 or 1 , depending on whether it is "workable" or "unworkable", respectively.

The pixel values of each image go through a normalization process. The normalization occurs to set all the pixel values to a number between zero and one. Normalization of pixels is an essential process, because it ensures a similar data distribution between input images. As a result, the $\mathrm{CNN}$ will converge faster during the training process.

In order to train a $\mathrm{CNN}$, all the images should have the same dimensions. Therefore, images must be resized to a dimension of 150 by 150 pixels. Figure 4 shows resized images that are now ready for the training process.

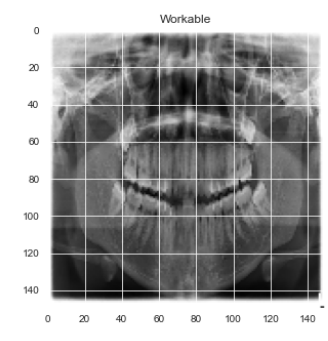

(a) Resized workable radiograph

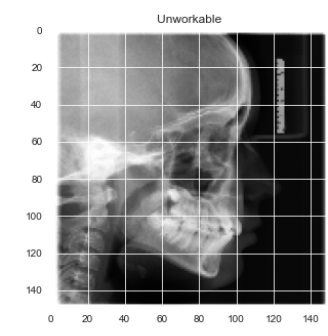

(b) Resized unworkable radiograph
Figure 4: Images must be resized to the same dimensions in order to train the $\mathrm{CNN}$.

A skew class distribution in the training dataset requires data augmentation to balance the classes. As seen in Figure 3a, there are significantly more "workable" than "unworkable' images'. In order to add more "unworkable" images to the dataset, various transformations must occur on the initial training dataset. The transformations used to generate more "unworkable" images included rotating images between $0^{\circ}$ and $180^{\circ}$, zooming into images by a factor of 0.2 , shifting images horizontally and vertically, and finally flipping images horizontally and vertically.

4.2.2 Configuration. An image classification problem has one input tensor and one output tensor at each layer. The tensor refers to the batch size combined with the image dimensions. A two dimensional array (the image) enters the layer, and a two dimensional array (image) leaves each layer. The $\mathrm{CNN}$ architecture is constructed with 14 layers in total. The first 10 alternate between the convolutional layer and the max-pooling layer. The convolutional layers extract the features to assist the machine with picking up specific characteristics of the image. The pooling layers act to reduce the image dimensionality without losing the features. Next, a flattening layer is used to flatten the input, without reducing the batch size. The flattening process is an essential step before applying the FLC, because it transforms the two-dimensional matrix of features (the image) into a vector form. Next, the FLCs are applied to generate a prediction. The sequential model represents the FLC as the "dense" layer. Finally, a "dropout" layer is added after each dense layer to help prevent overfitting. The dropout layer randomly sets input units to zero with a set frequency rate of 0.2 , to prevent overfitting, at each step during the training time.

Each convolutional layer is set to have a stride of one to reduce the spatial variance of the image. In addition, the convolutional layer uses a ReLU activation function to calculate the output of the neuron. The max-pooling layers have a stride of two to downsample the image, which in turn reduces the computational cost of running the machine learning process.

The image enters the first convolutional layer with a set dimension of $(150,150,1)$ and leaves at $(150,150,32)$. The depth on the image increases from 1 to 32 , meaning that the amount of colour information in each pixel increases. Thereafter, with each layer, the two-dimensional axes of the image continue to decrease while the depth continues to increase.

The number of parameters of each convolutional layer is a function of the image output multiplied by the input and the kernels. The image kernel refers to the dimensions of the pixels. The function follows a simple equation

parameters $=$ channels $_{\text {out }}\left(\right.$ channel $_{\text {in }_{n}} \cdot$ kernel $_{\text {width }} \cdot$ kernel $\left._{\text {height }}+1\right)$,

where "in channels" is the depth of the input image, "out channels" is the depth of the output image, the "kernel sizes" are equal and specify the width and height of the convolutional window, and the number one represents the bias.

Similarly, the number of parameters in the fully connected layers are

$$
\text { parameters }=\text { size }_{\text {out }}\left(\text { size }_{\text {in }}+1\right),
$$

where the in and out sizes represent the number of vector features along with a bias of 1 .

The first fully connected layer uses a ReLU activation function, whereas the final fully connected layer uses a sigmoid function to make the prediction.

4.2.3 Training process. Once the model has been configured, the training process can begin. The training dataset is fitted by using the image array as a learning input which has an associated output. The batch size for the training process is set to 32 , which refers to the number of training samples utilized in each iteration. In conjunction, 10 epochs are run. This hyperparameter defines the number of times that the learning algorithm works through the entire training set. In layman's terms, 32 different images will be used 10 times when training the model.

\subsection{Evaluation of results}

Overfitting is one of the main network design aspects to avoid when training a machine learning model. Overfitting refers to when the model fits the training data well, but cannot make accurate predictions and cannot generalize on new data. The performance of the model is assessed based on two metrics, namely accuracy and loss. Figure 5 plots the two metrics over each epoch in the training process.

Figure 5a displays the training and validation accuracy over each epoch. The accuracy of the model refers to correctly predicted 


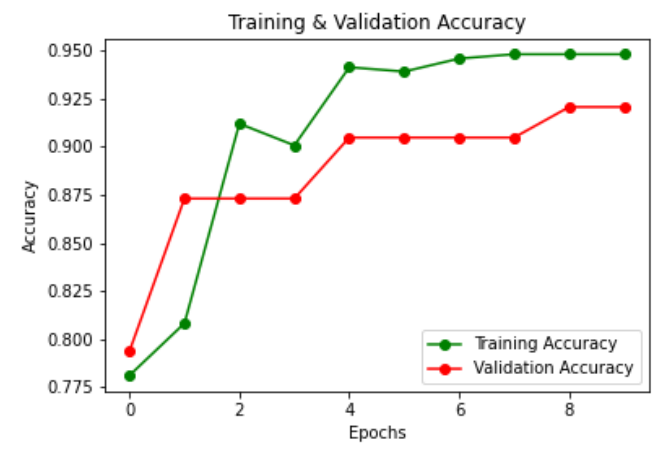

(a) A graph showing the accuracy over each epoch

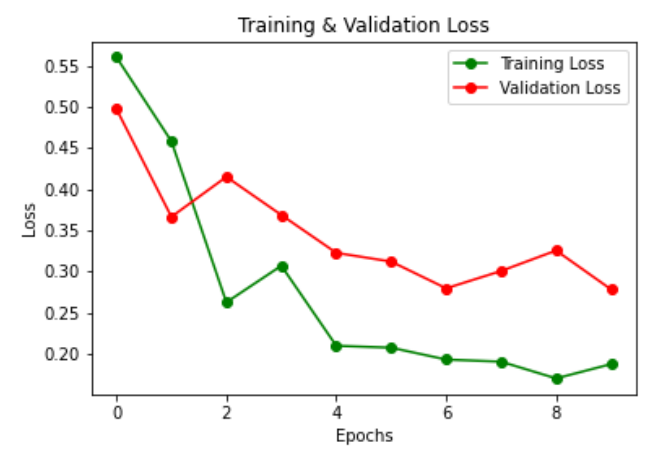

(b) A graph showing the loss over each epoch

Figure 5: Graphs comparing the training and validation performance of the model.

labels. Although the training accuracy is high here, the important metric is the validation accuracy. The graphs show that by the tenth epoch, the model has attained a $92.06 \%$ accuracy. The two graphs also start to flatten out, which means that more epochs may result in overfitting.

Figure $5 \mathrm{~b}$ displays the training and validation loss over each epoch. The ratio between training and validation loss indicates whether the model is overfitting, underfitting or working optimally. If the training loss is greater than the validation loss, the model is overfitting. Conversely, if the training loss is less than the validation loss, the model is underfitting. In order for the model to be satisfactory, the two metrics should be as close to each other as possible. The results from the tenth epoch of the trained model showed that training loss was 0.1873 , whereas the validation loss was 0.2782 . Although the model could be deemed to be underfitting, the metrics are close enough to each other to conclude them as satisfactory.

Table 1 shows the confusion matrix from the validation dataset. The table confirms the accuracy of the model by evaluating a strong true positive, and negative classification. Only eight images (6.2\%) were incorrectly labelled.
Table 1: Confusion matrix of the image classification algorithm

\begin{tabular}{l|c|c} 
& Workable & Unworkable \\
\hline Workable & 101 & 0 \\
\hline Unworkable & 8 & 20 \\
\hline
\end{tabular}

Table 2 shows important evaluation metrics that must be analyzed to confirm the performance of the model further. The precision determines out of the results classified as positive, how many were actually positive. The recall determines how many true positives were found by the model. The F1-score takes into account both the precision and recall as a weighted average. The F1-score is an excellent way to evaluate the overall performance of the model. The results from Table 2 once again convey a well-trained model. The F1-score for "workable" images is close to 1, which means that the model is confident in predicting "workable" images.

Table 2: The evaluation metrics of the neural network.

\begin{tabular}{|l|l|l|l|l|}
\hline & Precision & Recall & F1-score & Support \\
\hline Workable (Class 0) & 0.93 & 1.00 & 0.96 & 101 \\
\hline Unworkable (Class 1) & 1.00 & 0.71 & 0.83 & 28 \\
\hline Accuracy & & & 0.94 & 129 \\
\hline
\end{tabular}

\section{CONCLUSIONS}

The human mouth is a very complex organ and correctly diagnosing problems for treatment can be extremely time consuming for radiologists and dentists. Many different aspects, such as anomalies, have to be considered in dental diagnostics. In South Africa, dental screening centres are overloaded with patients and understaffed. Consequently, many patients leave undiagnosed or not having their dental X-rays adequately analyzed.

This paper developed a convolutional neural network $(\mathrm{CNN})$ to predict whether a captured dentiofacial radiograph is a panoramic radiograph and if this radiograph is of sufficient quality for further automated analysis. X-rays that are predicted as "unworkable" are then passed on for manual inspection. The image classification model was successfully able to detect "unworkable" images from the dataset. The model achieved an accuracy of $92.06 \%$.

Future work will consider approaches to further increase the accuracy, specifically working towards reducing the false positives. In addition, the next phase in the automated analysis of panoramic radiographs to detect teeth anomalies will be developed.

\section{REFERENCES}

[1] Charu C. Aggarwal. 2018. Neural Networks and Deep Learning. Springer International Publishing. 315-371 pages. https://doi.org/10.1007/978-3-319-94463-0

[2] Judit Forrai. 2007. History of x-ray in dentistry. Journal of Dental Clinics and Research 3, 3 (2007), 205-211.

[3] Debashis Ganguly, Srabonti Chakraborty, and Tai-hoon Kim. 2010. A Cognitive Study on Medical Imaging. International fournal of Bio-Science and Bio-Technology 2, 3 (2010), 1-18. 